\title{
Radiation-induced vertebral compression fracture following spine stereotactic radiosurgery: clinicopathological correlation
}

\author{
Report of 2 cases
}

\author{
Ameen Al-Omair, M.D., ${ }^{1}$ Roger Smith, M.D., ${ }^{2}$ Tim-Rasmus Kiehl, M.D.,${ }^{3,4}$ \\ Louis LaO, M.B.Ch.B., ${ }^{1}$ Eugene Yu, M.D., ${ }^{5}$ Eric M. MassicotTe, M.D., ${ }^{6}$ \\ Julia Keith, M.D., ${ }^{4,7}$ Michael G. Fehlings, M.D., Ph.D., ${ }^{6}$ and Arjun Sahgal, M.D. ${ }^{1,8}$ \\ ${ }^{1}$ Department of Radiation Oncology, Princess Margaret Hospital, University of Toronto; ${ }^{2}$ Department of \\ Radiology and ${ }^{6}$ Division of Neurosurgery and Spinal Program, Toronto Western Hospital, University of \\ Toronto; ${ }^{3}$ Department of Pathology, University Health Network; ${ }^{4}$ Department of Laboratory Medicine and \\ Pathobiology, University of Toronto; ${ }^{5}$ Department of Radiology and Otolaryngology-Head and Neck \\ Surgery, University Health Network, Mount Sinai Hospital and Women's College Hospital; and Departments \\ of ${ }^{7}$ Anatomical Pathology and ${ }^{8}$ Radiation Oncology, Sunnybrook Health Sciences Centre, University of \\ Toronto, Ontario, Canada
}

\begin{abstract}
Spine stereotactic radiosurgery (SRS) is increasingly being used to treat metastatic spinal tumors. As the experience matures, high rates of vertebral compression fracture (VCF) are being observed. What is unknown is the mechanism of action; it has been postulated but not confirmed that radiation itself is a contributing factor. This case report describes 2 patients who were treated with spine SRS who subsequently developed signal changes on MRI consistent with tumor progression and VCF; however, biopsy confirmed a diagnosis of radiation-induced necrosis in 1 patient and fibrosis in the other. Radionecrosis is a rare and serious side effect of high-dose radiation therapy and represents a diagnostic challenge, as the authors have learned from years of experience with brain SRS. These cases highlight the issues in the new era of spine SRS with respect to relying on imaging alone as a means of determining true tumor progression. In those scenarios in which it is unclear based on imaging if true tumor progression has occurred, the authors recommend biopsy to rule out radiation-induced effects within the bone prior to initiating salvage therapies. (http://thejns.org/doi/abs/10.3171/2013.2.SPINE12739)
\end{abstract}

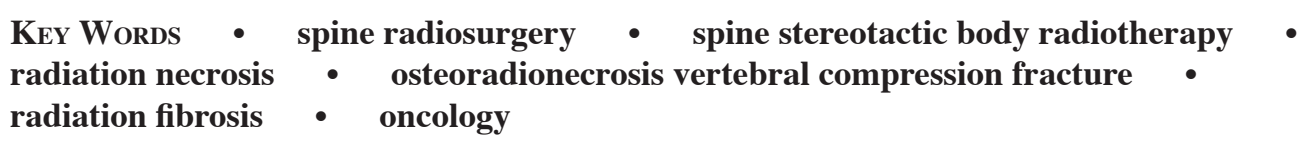

$\mathrm{S}$ PINE SRS, also known as stereotactic body radiotherapy, is increasingly being applied to treat primary and metastatic spinal tumors. ${ }^{12,13}$ Spine SRS is a paradigm shift from conventional radiotherapy practice, where the aim is to deliver low dose-per-fraction treatments (1.8-4.0 Gy per day) daily to a total dose limited by the tolerance of the spinal cord. Spine SRS overcomes this convention by using modern radiation technologies that allow for concentrating the dose within the tumor and away from the spinal cord, to permit tumor dose escalation while maintaining safe spinal cord dose

\footnotetext{
Abbreviations used in this paper: SINS = Spine Instability Neoplastic Score; SRS = stereotactic radiosurgery; VB = vertebral body; $\mathrm{VCF}=$ vertebral compression fracture .
}

constraints. ${ }^{5}$ Delivery precision has been reported to be within $1.5 \mathrm{~mm}$ and $1^{\circ}$ with a $95 \%$ confidence interval. ${ }^{7}$

Recently, the Canadian Association of Radiation Oncology defined stereotactic body radiotherapy (including as it pertains to the spine) as "The precise delivery of highly conformal and image-guided hypofractionated external beam radiotherapy, delivered in a single or few fraction(s), to an extracranial body target with doses at least biologically equivalent to a radical course when given over a protracted conventionally fractionated schedule."17 Philosophically, this translates to delivering a "locally curative" dose as opposed to a "locally palliative" dose for spinal metastatic disease. As experience with high dose-per-fraction spine SRS is gained (such that the most common practices range from 30 to $40 \mathrm{~Gy}$ in 5 frac- 


\section{Radiation necrosis and fracture following spine radiosurgery}

tions, 24 to 30 Gy in 2-3 fractions, and 16 to $24 \mathrm{~Gy}$ in a single fraction), ${ }^{8,12,14}$ we are learning that although we can achieve high rates of local tumor control, the costs include bone and spinal cord toxicity. $1,2,11,15,16,18$

Our group at the University of Toronto recently reported rates of post-SRS $\mathrm{VCF}^{2}$ We reported an actuarial $\mathrm{VCF}$ rate of $12.7 \%$ at 1 year post-SRS with a mean time to VCF of 3 months. It was observed that patients treated at the highest biologically effective dose level of 20-24 Gy in 1 fraction were the most susceptible to VCF. The mechanism of action was postulated as due to radiationinduced late effects within the tumor and bone tissue that ultimately weaken the VB to manifest clinically as VCF. In this report, we provide evidence to support this proposed mechanism of action by describing 2 cases of VCF post-SRS with histopathological findings confirming a diagnosis of radiation-induced necrosis in the first case and radiation-induced fibrosis in the second, as opposed to active tumor progression. Importantly, these 2 cases highlight the pitfalls of MRI in determining post-SRS tumor progression; without tissue confirmation these 2 cases would have been considered as local failures, placing the patient at risk for unnecessary surgical salvage procedures, repeat radiation, or changes in systemic therapy.

\section{Case Reports}

\section{Case 1}

This 51-year-old woman was diagnosed 4 years earlier with Stage IIIA adenocarcinoma of the lung and was treated with neoadjuvant concurrent chemotherapy and radiotherapy followed by surgery and adjuvant postoperative chemotherapy and radiation therapy. Two years later she presented with isolated nonmechanical low-back pain without neurological findings. Computed tomography scanning of the spine indicated an isolated mixed osteolytic/blastic bone lesion occupying the L-2 VB, presence of a mild VCF, and 1-2 mm of posterior bony retropulsion. The subsequent spine non-contrast enhanced MR image confirmed the L-2 VB metastases as the only site of spinal disease and demonstrated disease extending into the base of the left pedicle with 1-2 mm of anterior epidural disease extension (Fig. 1A and B). Her baseline total SINS $^{4}$ was 7, which categorized her spine as potentially unstable (Table 1). The patient was treated with spine SRS with a dose of 20 Gy in a single fraction (Fig. 1C and D). After spine SRS she was pain free, and she was subsequently treated with gefitinib. Follow-up non-contrast enhanced MRI indicated mild progression of the VCF at 6 months, which was asymptomatic, and a minor increase in T1 intermediate signal change with further dark signal foci (Fig. 1E). However, 2 years after spine SRS she complained of mild low-back pain again, without neurological findings. Computed tomography scanning of the spine showed significant progression of the VCF, and non-contrast enhanced MRI indicated a marked increased in the T1 intermediate signal change with diffuse darkening of the signal within the posterior half of the VB and minor posterior bony retropulsion (Fig. 1F). The T2-weighted MR images did not show any significant changes in signal intensity (images not shown). Clinically her disease was otherwise controlled with no new metastatic disease. Biopsy and simultaneous Kyphon Balloon Kyphoplasty (Medtronic) was performed. Histopathological analysis (Fig. 2A and B) showed abundant avital bone and necrotic debris with numerous minute fragments of metastatic adenocarcinoma interspersed between fragments of bone and necrotic tissue. Immunohistochemical studies (not shown) revealed that tumor cells were positive for CK7 and TTF1 but negative for CK20, which is consistent with pulmonary primary adenocarcinoma. Based on these findings, the patient was observed and continues to have stable disease 4 months postbiopsy with no evidence of disease progression.

\section{Case 2}

This 53-year-old man was diagnosed with metastatic neuroendocrine cancer with disease isolated to multiple segments of the spine. Initial treatment consisted of chemotherapy with 6 cycles of cisplatin and etoposide. Ten months after chemotherapy, he presented with nonmechanical low-back pain without neurological findings. Non-contrast enhanced T1-weighted MRI, T2-weighted MRI, and CT scanning of the spine confirmed disease progression within the L-3 and L-4 VBs. The baseline non-contrast enhanced MR images showed geographic foci of signal change on the T1- and T2-weighted images (Fig. 3A and B). The lesions were osteoblastic. The patient was treated with spine SRS to both vertebrae with a total dose of $24 \mathrm{~Gy}$ in 2 fractions (Fig. 3C). His baseline total SINS was 3 for each vertebra treated, which categorized his spine as stable (Table 1). At 1-year post-SRS, the noncontrast enhanced MR image indicated stable disease with no change in either T1 or T2 signal (Fig. 3D and E), and the patient underwent further chemotherapy. However, 3 years following spine SRS the patient complained of recurrent nonmechanical low-back pain. The non-contrast enhanced MR images indicated VCF isolated to L-3 but not L-4 (Fig. 3F and G). The intermediate T1 signal was diffusely increased throughout the VB within L-3, and T2 dark signal foci were also observed. No change was observed in the L-4 (treated) VB. With stable disease otherwise, we elected to perform a biopsy and Kyphon Balloon Kyphoplasty on the L-3 vertebra. Histopathological analysis showed markedly thinned bone trabeculae, and the bone marrow was completely replaced by either dense fibrous tissue with focal lymphocytic inflammation or loose paucicellular fibrous tissue (Fig. 2C and D). There was no evidence of residual carcinoma or necrosis. We have since observed the patient with no evidence of disease recurrence, now 6 months postbiopsy.

\section{Discussion}

This report is the first describing histopathological features of VCF after SRS by examining biopsy specimens in 2 cases that were otherwise considered radiographically as local tumor progression. We describe biopsy-confirmed radiation-induced necrosis and fibrosis, yielding imaging changes similar to tumor progression, and we propose its role in the pathogenesis of SRS-induced VCF. The ability to rely on imaging alone to determine the response after 
A. Al-Omair et al.

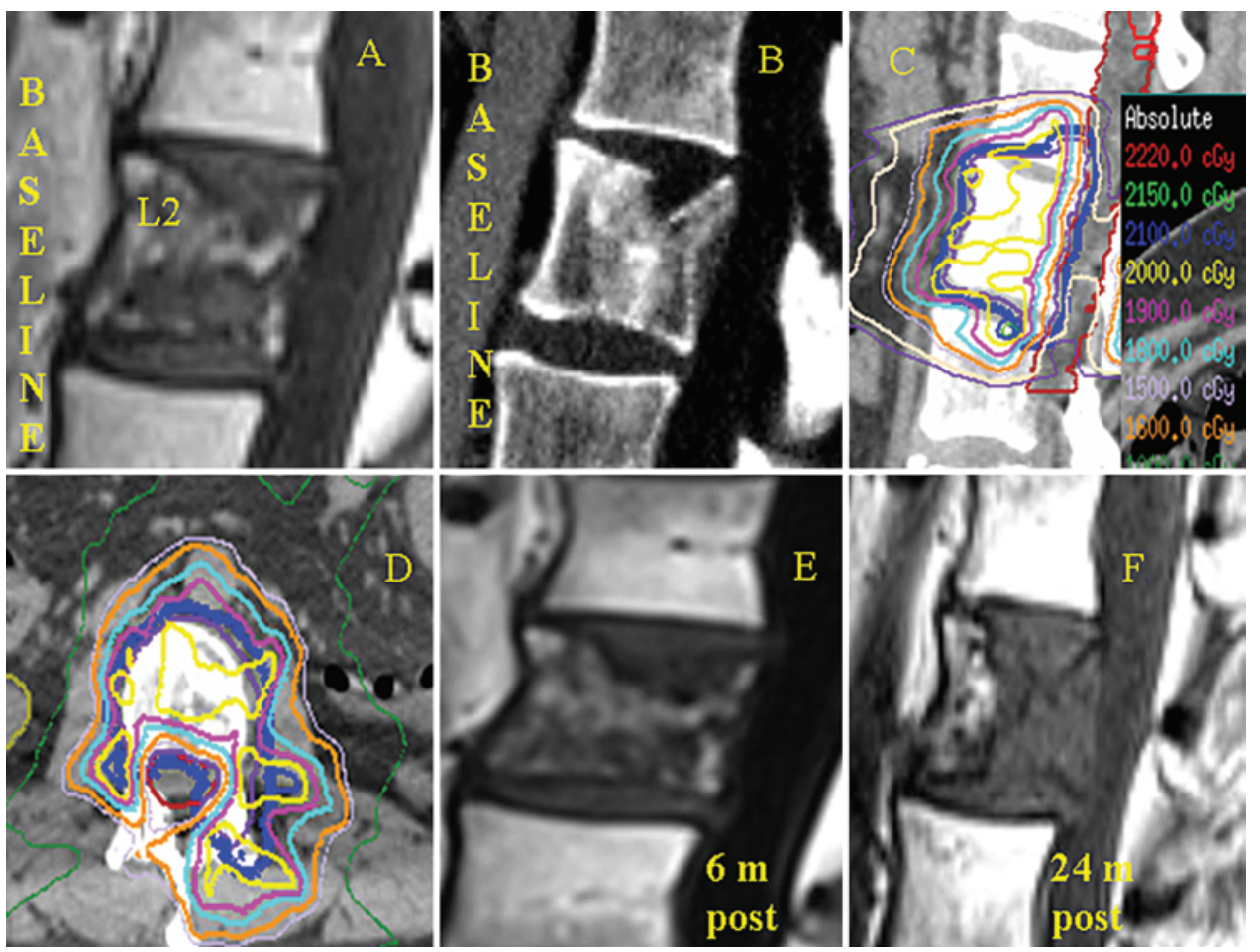

FIG. 1. Case 1. A: Baseline pre-SRS sagittal T1-weighted non-contrast enhanced MR image demonstrating tumor within the L-2 VB, based on the presence of abnormal intermediate T1 signal change, and presence of VCF. B: Baseline pre-SRS sagittal CT scan demonstrating a mixed osteolytic/blastic lesion occupying the L-2 VB and mild superior endplate compression fracture. C and D: Treatment-planning CT scans with representative isodose lines. The prescription dose was $20 \mathrm{~Gy}$ in a single fraction, with a maximum point dose within the thecal sac of $15.5 \mathrm{~Gy}$. E: Sagittal T1-weighted non-contrast enhanced MR image obtained 6 months $(\mathrm{m})$ after spine SRS, demonstrating progressive VCF within L-2 and no significant T1 signal change. F: Sagittal T1-weighted non-contrast enhanced MR image obtained 24 months after spine SRS, demonstrating further progression of the VCF, increased T1-weighted intermediate signal change, and posterior displacement of the bone toward the spinal canal.

spine SRS is going to be an increasing problem that will need further study and consensus, given that high-dose spine SRS has recently been associated with a significantly high rate of VCF.1,2,11 The Memorial Sloan-Kettering Cancer Center was the first to report on this complication with $39 \%$ of 71 treated vertebrae fracturing. ${ }^{11}$ Importantly, in that report the authors exclusively used high-dose singlefraction SRS (median total dose 24 Gy). The MD Anderson Cancer Center then reported fracture in $20 \%$ of their 123 vertebrae, and their SRS dosing practice varied from single to multiple fractions. ${ }^{1}$ The most recent analysis of post-SRS VCF was reported by our group, where $11 \%$ of 167 treated vertebrae fractured, yielding a 1-year actuarial fracture-free probability of $87.3 \%$. Similar to the MD Anderson Cancer Center experience, tumors were treated with various SRS dosing schemes (single and multiple fractions) as opposed to only single-fraction SRS. In that analysis, we also scored each treated vertebral segment according to SINS criteria ${ }^{2,4,6}$ (Table 1), and we analyzed these and additional dosimetric parameters to determine predictors of VCF. We observed that patients with lytic tumors, kyphosis/scoliosis, metastases from lung and hepatocellular primary tumors, and treatment with high-dose SRS with a dose per fraction of $20 \mathrm{~Gy}$ or more were at greatest risk for VCF. It was postulated that late radiation effects in the form of bone and tumor necrosis were likely a causative factor in destabilizing the vertebrae that resulted in the observed VCF. This is not implausible given that we have learned from the extensive experience with brain SRS that the risk of tissue necrosis increases with high doseper-fraction radiation, and at similar doses to those currently being applied to the spine. ${ }^{20}$ In this report, we have provided histopathological evidence to support our initial hypothesized mechanism of action.

In our first case we observed the presence of radiation-induced necrosis with scattered small clusters of adenocarcinoma consistent with the patient's original lung cancer (Figs. 1, 2A, and 2B). With this diagnosis, we decided to observe the patient as further radiation therapy could be catastrophic to the bone tissue, leading to frank instability requiring a major surgical intervention, and we could put the patient at increased risk of radiation-induced neural damage by exposing the critical neural tissues to more radiation. ${ }^{16}$ At this time, the patient has not suffered MRI-confirmed disease progression 4 months postbiopsy, and she remains stable on a regimen of her original systemic therapy. We postulate in this case that radiation-induced osteoradionecrosis was the causative factor for VCF and not tumor progression. In the second case (Figs. 2C, 2D, and 3), we observed the presence of marked thinning of the bone trabeculae, focal inflammation, and fibrosis in the L-3 biopsy specimen. In this case, the radiation effects on the bone tissue and the inflammatory reaction were causative for VCF and 


\section{Radiation necrosis and fracture following spine radiosurgery}

TABLE 1: Scoring for Cases 1 and 2 according to the SINS*

\begin{tabular}{|c|c|c|c|c|c|}
\hline \multirow[b]{2}{*}{ SINS Component } & \multirow[b]{2}{*}{ Description } & \multirow{2}{*}{$\begin{array}{c}\text { SINS } \\
\text { Classification }\end{array}$} & \multirow{2}{*}{$\begin{array}{l}\text { Case } 1 \\
\text { L-2 VB }\end{array}$} & \multicolumn{2}{|c|}{ Case 2} \\
\hline & & & & L-3 VB & $L-4 V B$ \\
\hline \multirow[t]{4}{*}{ location } & $\begin{array}{l}\text { junctional (occiput-C2, C7-T2, T11-L1, } \\
\text { L5-S1) }\end{array}$ & 3 & & & \\
\hline & mobile spine (C3-6, L2-4) & 2 & 2 & 2 & 2 \\
\hline & semirigid (T3-10) & 1 & & & \\
\hline & rigid (S2-5) & 0 & & & \\
\hline \multirow[t]{3}{*}{ pain } & yes & 3 & & & \\
\hline & nonmechanical pain & 1 & 1 & 1 & 1 \\
\hline & pain-free lesion & 0 & & & \\
\hline \multirow[t]{3}{*}{ bone lesion } & lytic & 2 & & & \\
\hline & mixed (lytic/blastic) & 1 & 1 & & \\
\hline & blastic & 0 & & 0 & 0 \\
\hline \multirow[t]{3}{*}{ radiographic spinal alignment } & subluxation/translation & 4 & & & \\
\hline & de novo deformity (kyphosis/scoliosis) & 2 & & & \\
\hline & normal alignment & 0 & 0 & 0 & 0 \\
\hline \multirow[t]{4}{*}{ VB collapse } & $>50 \%$ collapse & 3 & & & \\
\hline & $<50 \%$ collapse & 2 & 2 & & \\
\hline & no collapse $w />50 \%$ body involved & 1 & & & \\
\hline & none of the above & 0 & & 0 & 0 \\
\hline \multirow{3}{*}{$\begin{array}{l}\text { posterolat involvement of } \\
\text { spinal elements }\end{array}$} & bilat & 3 & & & \\
\hline & unilat & 1 & 1 & & \\
\hline & none of the above & 0 & & 0 & 0 \\
\hline total score & & & 7 & 3 & 3 \\
\hline
\end{tabular}

* As described by Fisher et al. ${ }^{4}$

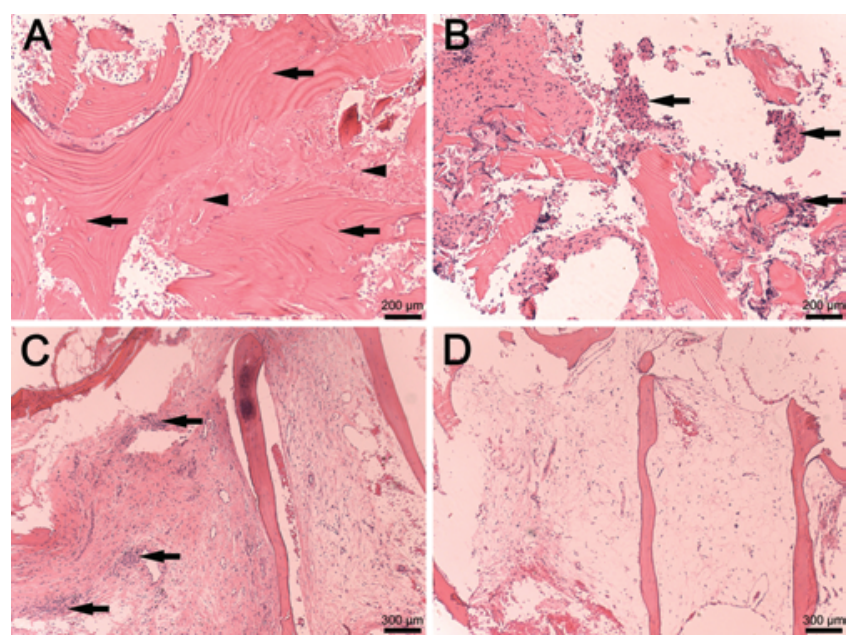

FIG. 2. Photomicrographs. A and B: Case 1. Section showing abundant avital bone (arrows) and necrotic debris (arrowheads, A). Section showing additional areas of numerous minute fragments of metastatic adenocarcinoma interspersed among fragments of bone and necrotic tissue (arrows, B). Immunohistochemical studies (not shown) revealed that the tumor cells were positive for CK7 and TTF1 but negative for CK20. C and D: Case 2. Sections showing markedly thinned bone trabeculae. The bone marrow is completely replaced by either dense fibrous tissue with focal lymphocytic inflammation (arrows, C) or loose paucicellular fibrous tissue (D). There was no evidence of carcinoma or necrosis. $H \& E$, original magnification $\times 10$. not tumor progression. Although we observed no overt necrotic tissue, clearly the bone had undergone radiationinduced degeneration (Fig. 2C and D). Importantly, it has been postulated that radiation-induced fibrosis is linked to the development and progression to radiation-induced osteoradionecrosis. ${ }^{23}$ Therefore, the histopathological finding of radiation-induced fibrosis supports the idea of radiation-induced late effects on the bone as a factor in the pathogenesis of VCF post-SRS.

Osteoradionecrosis is a rare but well-known late effect of high-dose radiation and has been reported predominantly in the mandible following high-dose conventional radiation therapy in the head and neck literature. ${ }^{9}$ Osteoradionecrosis has been defined as a slow-healing radiation-induced ischemic necrosis of the bone tissue. ${ }^{23}$ The mechanism of osteoradionecrosis is thought to relate to radiation therapy by the production of a hypoxic, hypocellular, and hypovascular environment in which the basic metabolic demands for cellular survival cannot be met. ${ }^{23}$ The onset of osteoradionecrosis has been described to range from 6 months to 3 years posttreatment, and even up to 25 years after radiation therapy..$^{10}$ With respect to the spine, there have been few specific reports of osteoradionecrosis, ${ }^{3,10,19,21}$ and ours is the first report specific to spine SRS. Ultimately, the histopathological analysis of these 2 cases supports our previously postulated mechanism of action that high-dose radiation is a factor in develop- 
A. Al-Omair et al.

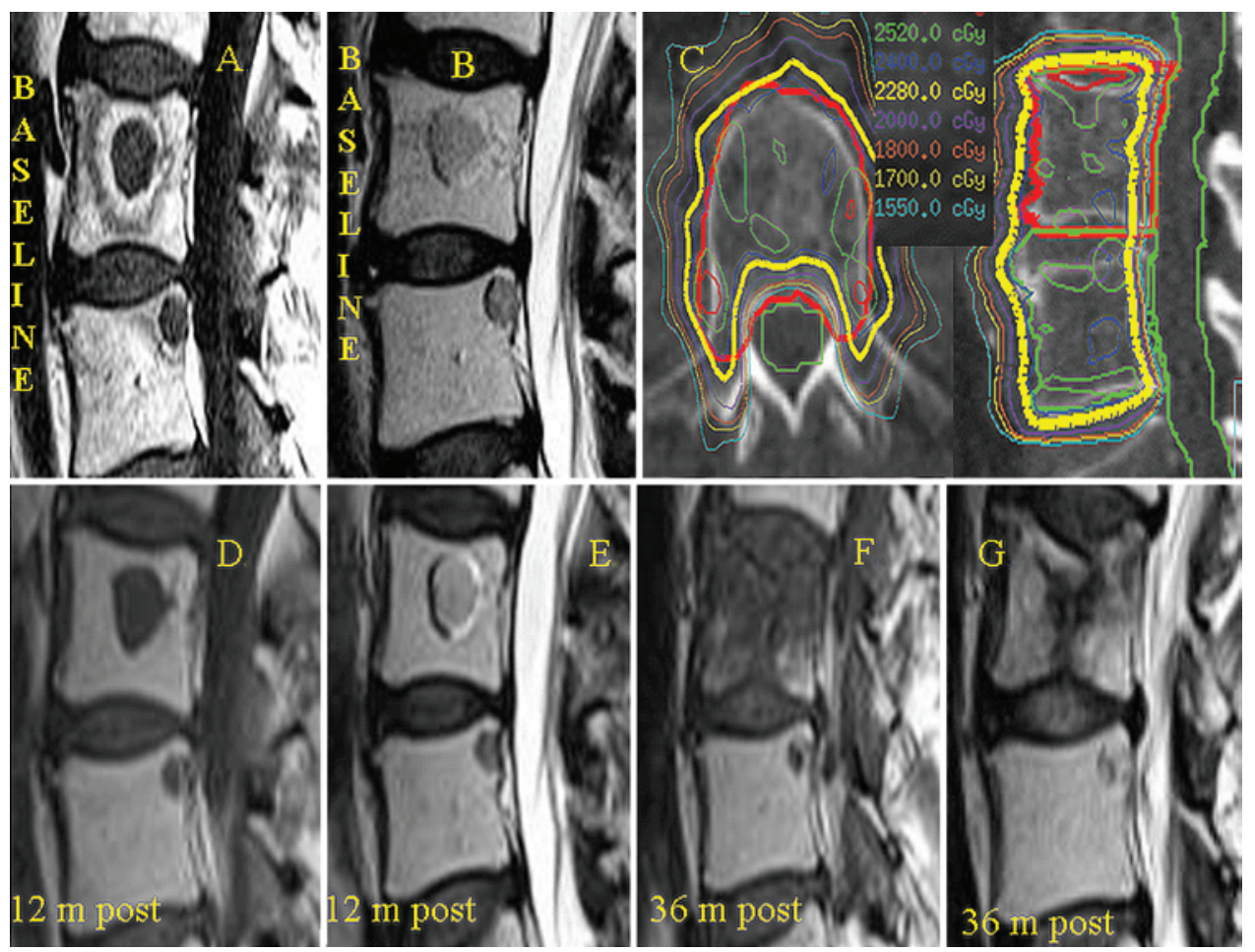

FIG. 3. Case 2. A and B: Sagittal non-contrast enhanced T1- (A) and T2- (B) weighted MR images obtained prior to spine SRS, demonstrating metastatic foci within the L-3 and L-4 VBs. C: Planning CT scans with isodose lines as indicated. Both L-3 and L-4 were treated with $24 \mathrm{~Gy}$ in 2 fractions, and the thecal sac maximum point dose was $14.3 \mathrm{~Gy}$. D and E: Sagittal noncontrast enhanced T1- (D) and T2- (E) weighted MR images obtained 12 months after spine SRS, demonstrating stability of the L-3 and L-4 lesions with no signal change compared with the baseline images. F and G: Sagittal non-contrast enhanced T1$(F)$ and T2- $(G)$ weighted MR images obtained 36 months after spine SRS, showing possible progression of the bony metastatic disease within the L-3 VB given the increase in the T1 intermediate signal, mild compression fracture, and loss of height without extraosseous soft-tissue tumor extension. The L-4 lesion remained stable with no signal change on non-contrast enhanced T1or T2-weighted MR images, and there was no compression fracture.

ing VCF post-SRS, as opposed to strictly anatomical or tumor-related structural factors. The findings from these 2 cases also support the role of biopsy when it is unclear by imaging alone that disease progression has occurred.

The issue of how to determine disease progression post-SRS is a major problem that needs clarification. There are no guidelines or consensus criteria on this issue. The description of the follow-up MR images in these 2 cases, as illustrated in Figs. 1 and 3, highlights the challenges of using conventional MRI as a means of response monitoring. In both cases, the radiologist reported disease progression based on the increased T1 signal change, visualized as darkening of the normal marrow signal within the VB, which is the preferred MRI sequence to visualize tumor within bone. If biopsy had not been performed in these 2 patients, they may have undergone unnecessary further radiation therapy, major spine surgery, or initiated/changed systemic chemotherapy, as opposed to a minimally invasive stabilization procedure (Kyphon Balloon Kyphoplasty in these cases) and observation. As yet, there are no clear guidelines as to the use of biological imaging such as PET scanning or other MRI sequences that can improve the ability to determine true disease progression from radiation-induced imaging effects. ${ }^{22}$ This has been a long-standing problem in the brain with years of research that has yet to solve this problem. ${ }^{22}$ Of note, in the brain the addition of Gd contrast is also not useful since necrotic tissue enhances in a similar way to tumor; however, the normal brain tissue does not enhance. This is unlike the spine where the normal marrow also enhances with contrast, which renders contrast-enhanced MRI unnecessary and explains why metastatic spine imaging is performed without contrast. Ultimately, the lesson is to perform a biopsy when it is questionable whether the disease has truly progressed. For example, if signal change is isolated to within the treated anatomy with no new epidural or paraspinal disease extension, then in these situations biopsy is recommended to rule out radiation-induced change.

Last, we note an interesting aspect in Case 2 (Fig. 3). In this patient the L-4 VB was treated concurrently with the L-3 VB as a single target and prescribed dose. The tumors within both the L-3 and L-4 VBs were well circumscribed and sclerotic; however, the tumor volume was smaller within the L-4 body (Fig. 3). No VCF was observed in the L-4 VB, unlike the VCF observed in L-3. Therefore, we postulate that both tumor effects and healthy bone effects from the high-dose radiation are likely interrelated in the mechanism of radiation-induced VCF, and further study is required to understand this relationship.

This study is limited by the fact that we only analyzed tissue within a biopsy specimen and that only 2 cases were analyzed. More cases are required to draw firm 


\section{Radiation necrosis and fracture following spine radiosurgery}

conclusions. However, this first report provides insight into the pathophysiology of radiation-induced VCF postSRS and expands on the challenges to response monitoring specific to spine SRS with conventional MRI. Our future study includes a comprehensive analysis of each VCF case from our original series. ${ }^{2}$

\section{Conclusions}

We recommend biopsy when it is unclear based on imaging alone if true progression has occurred, as marrow signal change within SRS-treated bone by itself may not be an objective finding and may be a consequence of radiation-induced necrosis and fibrosis.

\section{Disclosure}

Drs. Sahgal and Smith have received honoraria from Medtronic Kyphoplasty for educational talks. Otherwise the authors have no other conflict of interest.

Author contributions to the study and manuscript preparation include the following. Conception and design: Sahgal, Al-Omair, Fehlings. Acquisition of data: Sahgal, Al-Omair, Smith, Kiehl, Lao, Yu, Massicotte. Analysis and interpretation of data: Sahgal, Al-Omair, Smith, Kiehl, Yu, Keith, Fehlings. Drafting the article: Sahgal, Al-Omair, Kiehl, Keith, Fehlings. Critically revising the article: all authors. Reviewed submitted version of manuscript: all authors. Approved the final version of the manuscript on behalf of all authors: Sahgal. Administrative/technical/material support: Sahgal. Study supervision: Sahgal.

\section{References}

1. Boehling NS, Grosshans DR, Allen PK, McAleer MF, Burton AW, Azeem S, et al: Vertebral compression fracture risk after stereotactic body radiotherapy for spinal metastases. Clinical article. J Neurosurg Spine 16:379-386, 2012

2. Cunha MV, Al-Omair A, Atenafu EG, Masucci GL, Letourneau D, Korol R, et al: Vertebral compression fracture (VCF) after spine stereotactic body radiation therapy (SBRT): analysis of predictive factors. Int J Radiat Oncol Biol Phys 84:e343e 349,2012

3. Donovan DJ, Huynh TV, Purdom EB, Johnson RE, Sniezek JC: Osteoradionecrosis of the cervical spine resulting from radiotherapy for primary head and neck malignancies: operative and nonoperative management. Case report. J Neurosurg Spine 3:159-164, 2005

4. Fisher CG, DiPaola CP, Ryken TC, Bilsky MH, Shaffrey CI, Berven SH, et al: A novel classification system for spinal instability in neoplastic disease: an evidence-based approach and expert consensus from the Spine Oncology Study Group. Spine (Phila Pa 1976) 35:E1221-E1229, 2010

5. Foote M, Letourneau D, Hyde D, Massicotte E, Rampersaud R, Fehlings M, et al: Technique for stereotactic body radiotherapy for spinal metastases. J Clin Neurosci 18:276-279, 2011

6. Fourney DR, Frangou EM, Ryken TC, Dipaola CP, Shaffrey CI, Berven SH, et al: Spinal instability neoplastic score: an analysis of reliability and validity from the spine oncology study group. J Clin Oncol 29:3072-3077, 2011

7. Hyde D, Lochray F, Korol R, Davidson M, Wong CS, Ma L, et al: Spine stereotactic body radiotherapy utilizing cone-beam CT image-guidance with a robotic couch: intrafraction motion analysis accounting for all six degrees of freedom. Int J Radiat Oncol Biol Phys 82:e555-e562, 2012

8. Masucci GL, Yu E, Ma L, Chang EL, Letourneau D, Lo S, et al: Stereotactic body radiotherapy is an effective treatment in reirradiating spinal metastases: current status and practical considerations for safe practice. Expert Rev Anticancer Ther 11:1923-1933, 2011
9. Nabil S, Samman N: Risk factors for osteoradionecrosis after head and neck radiation: a systematic review. Oral Surg Oral Med Oral Pathol Oral Radiol 113:54-69, 2012

10. Rolton DJ, Blagg SE, Hughes RJ: Osteoradionecrosis of the lumbar spine 25 years after radiotherapy. J Bone Joint Surg Br 93:1279-1281, 2011

11. Rose PS, Laufer I, Boland PJ, Hanover A, Bilsky MH, Yamada $\mathrm{J}$, et al: Risk of fracture after single fraction image-guided intensity-modulated radiation therapy to spinal metastases. J Clin Oncol 27:5075-5079, 2009

12. Sahgal A, Bilsky M, Chang EL, Ma L, Yamada Y, Rhines LD, et al: Stereotactic body radiotherapy for spinal metastases: current status, with a focus on its application in the postoperative patient. A review. J Neurosurg Spine 14:151-166, 2011

13. Sahgal A, Chou D, Ames C, Ma L, Lamborn K, Huang K, et al: Image-guided robotic stereotactic body radiotherapy for benign spinal tumors: the University of California San Francisco preliminary experience. Technol Cancer Res Treat 6:595-604, 2007

14. Sahgal A, Larson DA, Chang EL: Stereotactic body radiosurgery for spinal metastases: a critical review. Int J Radiat Oncol Biol Phys 71:652-665, 2008

15. Sahgal A, Ma L, Gibbs I, Gerszten PC, Ryu S, Soltys S, et al: Spinal cord tolerance for stereotactic body radiotherapy. Int J Radiat Oncol Biol Phys 77:548-553, 2010

16. Sahgal A, Ma L, Weinberg V, Gibbs IC, Chao S, Chang UK, et al: Reirradiation human spinal cord tolerance for stereotactic body radiotherapy. Int J Radiat Oncol Biol Phys 82:107-116, 2012

17. Sahgal A, Roberge D, Schellenberg D, Purdie TG, Swaminath A, Pantarotto J, et al: The Canadian Association of Radiation Oncology scope of practice guidelines for lung, liver and spine stereotactic body radiotherapy. Clin Oncol (R Coll Radiol) 24:629-639, 2012

18. Sahgal A, Weinberg V, Ma L, Chang E, Chao S, Muacevic A, et al: Probabilities of radiation myelopathy specific to stereotactic body radiation therapy to guide safe practice. Int J Radiat Oncol Biol Phys 85:341-347, 2013

19. Sato Y, Uematsu H, Yoshida M, Shioura H, Muramatsu J, Kimura $\mathrm{H}$, et al: Osteonecrosis induced by intraoperative radiotherapy for pancreatic cancer. Eur Radiol 16:242-243, 2006

20. Shaw E, Scott C, Souhami L, Dinapoli R, Kline R, Loeffler J, et al: Single dose radiosurgical treatment of recurrent previously irradiated primary brain tumors and brain metastases: final report of RTOG protocol 90-05. Int J Radiat Oncol Biol Phys 47:291-298, 2000

21. Smith VA, Lentsch EJ: Life-threatening cervical spine collapse as a result of postradiation osteonecrosis-case report and review of the literature. Head Neck [epub ahead of print], 2012

22. Stockham AL, Tievsky AL, Koyfman SA, Reddy CA, Suh JH, Vogelbaum MA, et al: Conventional MRI does not reliably distinguish radiation necrosis from tumor recurrence after stereotactic radiosurgery. J Neurooncol 109:149-158, 2012

23. Zhuang Q, Zhang Z, Fu H, He J, He Y: Does radiation-induced fibrosis have an important role in pathophysiology of the osteoradionecrosis of jaw? Med Hypotheses 77:63-65, 2011

\section{Manuscript submitted August 7, 2012.}

Accepted February 7, 2013.

Please include this information when citing this paper: published online March 15, 2013; DOI: 10.3171/2013.2.SPINE12739.

Address correspondence to: Arjun Sahgal, M.D., Department of Radiation Oncology, Sunnybrook Health Sciences Centre and Princess Margaret Hospital, University of Toronto, 610 University Avenue, Toronto, Ontario M6G 2M5, Canada. email: arjun. sahgal@rmp.uhn.on.ca. 